\title{
On Super Edge-Connectivity of Cartesian Product Graphs
}

\author{
Min Lü and Guo-Liang Chen \\ Anhui Province-Most Key Co-Lab of High Performance Computing and Its Applications, \\ Department of Computer Science and Technology, University of Science and Technology of China, \\ Hefei, Anhui, 230026, People's Republic of China
}

\author{
Jun-Ming Xu \\ Department of Mathematics, University of Science and Technology of China, Hefei, Anhui, 230026, \\ People's Republic of China
}

\begin{abstract}
The super edge-connectivity $\lambda^{\prime}$ of a connected graph $G$ is the minimum cardinality of an edge-cut $F$ in $G$ such that every component of $G-F$ contains at least two vertices. Let $G_{i}$ be a connected graph with order $n_{i}$, minimum degree $\delta_{i}$ and edge-connectivity $\lambda_{i}$ for $i=1,2$. This article shows that $\lambda^{\prime}\left(G_{1} \times G_{2}\right) \geq \min \left\{n_{1} \lambda_{2}, n_{2} \lambda_{1}, \lambda_{1}+\right.$ $\left.2 \lambda_{2}, 2 \lambda_{1}+\lambda_{2}\right\}$ for $n_{1}, n_{2} \geq 3$ and $\lambda^{\prime}\left(K_{2} \times G_{2}\right)=\min \left\{n_{2}, 2 \lambda_{2}\right\}$, which generalizes the main result of Shieh on the super edge-connectedness of the Cartesian product of two regular graphs with maximum edge-connectivity. In particular, this article determines $\lambda^{\prime}\left(G_{1} \times G_{2}\right)=\min \left\{n_{1} \delta_{2}\right.$, $\left.n_{2} \delta_{1}, \xi\left(G_{1} \times G_{2}\right)\right\}$ if $\lambda^{\prime}\left(G_{i}\right)=\xi\left(G_{i}\right)$, where $\xi(G)$ is the minimum edge-degree of a graph G. (c) 2006 Wiley Periodicals, Inc. NETWORKS, Vol. 49(2), 152-157 2007
\end{abstract}

Keywords: $\quad$ super edge-connectivity; Cartesian product; super- $\lambda$; $\lambda^{\prime}$-graph; $\lambda^{\prime}$-optimal graph

\section{INTRODUCTION}

Throughout this article, a graph $G=(V, E)$ always means a finite undirected graph without self-loops or multiple edges, where $V=V(G)$ is the vertex-set and $E=E(G)$ is the edgeset. For any edge $u v \in E$, the parameter $\xi_{G}(u v)=d_{G}(u)+$ $d_{G}(v)-2$ is the degree of the edge $u v$ and the parameter $\xi(G)=\min \left\{\xi_{G}(u v) \mid u v \in E\right\}$ is the minimum edge-degree of $G$. The symbols $K_{1, n-1}$ and $K_{n}$ denote a star graph and a complete graph with $n$ vertices, respectively. For the graph theoretical terminology and notation not defined here, we refer the reader to [13].

It is well known that when the underlying topology of an interconnection network is modeled by a connected graph

Received December 2005; accepted July 2006

Correspondence to: M. Lü; e-mail: 1vmin05@ustc.edu.cn

Contract grant sponsor: National Natural Science Foundation of China; Contract grant number: 60533020

DOI $10.1002 /$ net.20149

Published online in Wiley InterScience (www.interscience.wiley. com).

(C) 2006 Wiley Periodicals, Inc.
$G=(V, E)$, where $V$ is the set of processors and $E$ is the set of communication links in the network, the edge-connectivity $\lambda(G)$ of $G$ is an important measurement for the fault tolerance of the network. In general, the larger $\lambda(G)$ is, the more reliable the network is. It is well known that $\lambda(G) \leq \delta(G)$, where $\delta(G)$ is the minimum degree of $G$. A connected graph $G$ is said to be maximally edge-connected (in short, $\max -\lambda$ ) if $\lambda(G)=\delta(G)$. Obviously, the set of edges incident with a vertex of degree $\delta(G)$ is certainly a minimum edge-cut and isolates a vertex when $G$ is $\max -\lambda$.

A graph $G$ is said to be super edge-connected (in short, super- $\lambda$ ) if $G$ is max- $\lambda$ and every minimum edge-cut isolates a vertex of $G$.

It has been shown that a super- $\lambda$ network is the most reliable and has the smallest edge failure rate (see, e.g., $[17,18]$ ). Several sufficient conditions for a graph to be max- $\lambda$ or super- $\lambda$ have been given in the literature (see, e.g., [6]).

A quite natural problem is that if a connected graph $G$ is super- $\lambda$ then how many edges have to be removed to disconnect $G$ such that every component of the resulting graph contains no isolated vertices. This problem results in the concept of the super edge-connectivity, introduced first by Fiol et al. in [4].

An edge-cut $F$ is called a super edge-cut of $G$ if $G-$ $F$ contains no isolated vertices. In general, super edge-cuts do not always exist. The super edge-connectivity $\lambda^{\prime}(G)$ is the minimum cardinality of a super edge-cut in $G$ if super edge-cuts exist, and, by convention, is $+\infty$ otherwise.

The new parameter $\lambda^{\prime}$ in conjunction with $\lambda$ can provide more accurate measures for the fault tolerance of a largescale parallel processing system and, thus, has received the attention of many researchers in recent years (see, e.g., [3-9, $11,14-16]$ ). Esfahanian and Hakimi [3] showed that if $G$ is neither $K_{1, n-1}$ nor $K_{3}$, then

$$
\lambda(G) \leq \lambda^{\prime}(G) \leq \xi(G) .
$$

A connected graph $G$ is called a $\lambda^{\prime}$-graph if $G$ is neither $K_{1, n-1}$ nor $K_{3}$. It is easy to see that if $\lambda^{\prime}(G)>\lambda(G)$ then $G$ 
is super- $\lambda$. A super- $\lambda$ graph $G$ is said to be optimally super edge-connected (in short, $\lambda^{\prime}$-optimal) if $\lambda^{\prime}(G)=\xi(G)$.

Recently, Chiue and Shieh [1] have given some sufficient conditions for the Cartesian product $G_{1} \times G_{2}$ to be super- $\lambda$; Shieh [10] has proved that $G_{1} \times G_{2}$ is super- $\lambda$ if both $G_{1}$ and $G_{2}$ are regular and max- $\lambda$ except for $K_{2} \times K_{n}$, where $n \geq 2$. Ueffing and Volkmann [11] have investigated the $\lambda^{\prime}$-optimality of $G_{1} \times G_{2}$ when both $G_{1}$ and $G_{2}$ are $\lambda^{\prime}$-optimal. Li and Xu [7] have determined $\lambda^{\prime}\left(K_{2} \times G\right)=$ $\min \left\{n, 2 \delta(G), 2 \lambda^{\prime}(G)\right\}$ for any connected graph $G$ with $n$ vertices.

Let $G_{i}$ be a connected graph of order $n_{i}$, minimum degree $\delta_{i}$ and edge-connectivity $\lambda_{i}$ for $i=1,2$. In this article, we show that $\lambda^{\prime}\left(G_{1} \times G_{2}\right) \geq \min \left\{n_{1} \lambda_{2}, n_{2} \lambda_{1}, \lambda_{1}+2 \lambda_{2}, 2 \lambda_{1}+\lambda_{2}\right\}$ for $n_{1}, n_{2} \geq 3$ by refining the technique of Chiue and Shieh in [1] and determine that $\lambda^{\prime}\left(K_{2} \times G_{2}\right)=\min \left\{n_{2}, 2 \lambda_{2}\right\}$, which generalizes the result of Shieh [10]. In particular, similar to the proof of Theorem 4.1 in [11], we determine that $\lambda^{\prime}\left(G_{1} \times\right.$ $\left.G_{2}\right)=\min \left\{n_{1} \delta_{2}, n_{2} \delta_{1}, \xi\left(G_{1} \times G_{2}\right)\right\}$ if both $G_{1}$ and $G_{2}$ are $\lambda^{\prime}$-optimal.

The proofs of these results are given in Section 3.

\section{PRELIMINARIES}

Let $G_{1}=\left(V_{1}, E_{1}\right)$ and $G_{2}=\left(V_{2}, E_{2}\right)$. The union of two graphs (not necessarily disjoint) $G_{1}$ and $G_{2}$, denoted by $G_{1} \cup$ $G_{2}$, is the graph with the vertex-set $V\left(G_{1} \cup G_{2}\right)=V_{1} \cup V_{2}$ and the edge-set $E\left(G_{1} \cup G_{2}\right)=E_{1} \cup E_{2}$. The Cartesian product of $G_{1}$ and $G_{2}$, denoted by $G_{1} \times G_{2}$, is the graph with the vertex-set $V_{1} \times V_{2}$ such that two vertices $\left(x_{1}, y_{1}\right)$ and $\left(x_{2}, y_{2}\right)$ are adjacent if and only if either $x_{1}=x_{2} \in V_{1}$ with $y_{1} y_{2} \in E_{2}$ or $y_{1}=y_{2} \in V_{2}$ with $x_{1} x_{2} \in E_{1}$.

By the definition of the Cartesian product $G=G_{1} \times G_{2}$, for any vertex $(x, y) \in V(G)$,

$$
d_{G}(x, y)=d_{G_{1}}(x)+d_{G_{2}}(y),
$$

and if $x_{1} x_{2} \in E_{1}$ or $y_{1} y_{2} \in E_{2}$, then

$$
\begin{aligned}
& \xi_{G}\left(\left(x_{1}, y_{1}\right)\left(x_{2}, y_{1}\right)\right)=\xi_{G_{1}}\left(x_{1} x_{2}\right)+2 d_{G_{2}}\left(y_{1}\right), \\
& \xi_{G}\left(\left(x_{1}, y_{1}\right)\left(x_{1}, y_{2}\right)\right)=\xi_{G_{2}}\left(y_{1} y_{2}\right)+2 d_{G_{1}}\left(x_{1}\right),
\end{aligned}
$$

respectively, and consequently,

$$
\xi(G)=\min \left\{\xi\left(G_{1}\right)+2 \delta\left(G_{2}\right), \xi\left(G_{2}\right)+2 \delta\left(G_{1}\right)\right\} .
$$

For convenience, we define two kinds of subgraphs $G_{1 y}$ and $G_{2 x}$ of $G_{1} \times G_{2}$ as follows.

$$
\begin{aligned}
& V\left(G_{1 y}\right)=\left\{(x, y) \mid x \in V_{1}\right\} \text { and } \\
& E\left(G_{1 y}\right)=\left\{\left(x_{1}, y\right)\left(x_{2}, y\right) \mid x_{1} x_{2} \in E_{1}\right\} \text { for any } y \in V_{2} ; \\
& V\left(G_{2 x}\right)=\left\{(x, y) \mid y \in V_{2}\right\} \text { and } \\
& E\left(G_{2 x}\right)=\left\{\left(x, y_{1}\right)\left(x, y_{2}\right) \mid y_{1} y_{2} \in E_{2}\right\} \text { for any } x \in V_{1} .
\end{aligned}
$$

It is clear that $G_{1 y}$ is isomorphic to $G_{1}$ for any $y \in V_{2}$ and $G_{2 x}$ is isomorphic to $G_{2}$ for any $x \in V_{1}$. Let

$V_{1 y}=V\left(G_{1 y}\right), E_{1 y}=E\left(G_{1 y}\right), V_{2 x}=V\left(G_{2 x}\right), E_{2 x}=E\left(G_{2 x}\right)$.
Then

$$
\begin{gathered}
E_{1 y} \cap E_{1 y^{\prime}}=\emptyset, \quad \text { for any } y, y^{\prime} \in V_{2}, y \neq y^{\prime} ; \\
E_{2 x} \cap E_{2 x^{\prime}}=\emptyset, \quad \text { for any } x, x^{\prime} \in V_{1}, x \neq x^{\prime} ; \\
V_{1 y} \cap V_{2 x}=\{(x, y)\}, \quad E_{1 y} \cap E_{2 x}=\emptyset \\
\quad \text { for any } x \in V_{1}, y \in V_{2} ; \\
E\left(G_{1} \times G_{2}\right)=\left(\cup_{y \in V_{2}} E_{1 y}\right) \cup\left(\cup_{x \in V_{1}} E_{2 x}\right) .
\end{gathered}
$$

To check whether a union graph is connected or not, the following concept and results, due to Chiue and Shieh [1], are useful.

Definition (Separability). For $G=G_{1} \cup G_{2} \cup \cdots \cup G_{k}$, $V(G)$ is called separable if and only if $V(G)$ can be partitioned into two disjoint nonempty sets $A$ and $A^{\prime}$ such that $A \cup A^{\prime}=V(G)$ and each $V\left(G_{i}\right)$ is a subset of either $A$ or $A^{\prime}$ for $i=1,2, \ldots, k$.

Lemma 1. Suppose $G=\cup_{i=1}^{k} G_{i}$, where $G_{i}$ is connected for $i=1,2, \ldots, k$. If $V(G)$ is nonseparable, then $G$ is connected.

Remark 1. Because $V_{1 y} \cap V_{2 x}=\{(x, y)\}, V_{1 y} \cup V_{2 x}$ is nonseparable for any $x \in V_{1}$ and $y \in V_{2}$.

\section{MAIN RESULTS}

We first introduce some notation used in this section. Let $G=(V, E)$ be a graph. For two disjoint nonempty subsets $X$ and $Y$ of $V$, denote $(X, Y)_{G}=\{x y \in E \mid x \in X, y \in Y\}$. If $Y=V \backslash X$, then we write $E_{G}(X)=(X, Y)_{G}$ and $d_{G}(X)=$ $\left|E_{G}(X)\right|$.

A super edge-cut $F$ of $G$ is called a $\lambda^{\prime}$-cut if $|F|=\lambda^{\prime}(G)$. It is clear that $G-F$ has exactly two components for any $\lambda^{\prime}$-cut $F$. A nonempty and proper subset $X$ of $V$ is called a $\lambda^{\prime}$-fragment of $G$ if $E_{G}(X)$ is a $\lambda^{\prime}$-cut of $G$. The minimum $\lambda^{\prime}$-fragment over all $\lambda^{\prime}$-fragments of $G$ is called a $\lambda^{\prime}$-atom of $G$.

For $F \subseteq E\left(G_{1} \times G_{2}\right)$, let

$$
\begin{aligned}
& G_{1 y}^{\prime}=G_{1 y}-F \text { for any } y \in V_{2}, \\
& G_{2 x}^{\prime}=G_{2 x}-F \text { for any } x \in V_{1} .
\end{aligned}
$$

Then, it is clear that

$$
\begin{array}{r}
V\left(G_{1 y}^{\prime}\right)=V_{1 y}, V\left(G_{2 x}^{\prime}\right)=V_{2 x} \text { for any } x \in V_{1} \text { and } y \in V_{2} ; \\
G_{1} \times G_{2}-F=\left(\cup_{y \in V_{2}} G_{1 y}^{\prime}\right) \cup\left(\cup_{x \in V_{1}} G_{2 x}^{\prime}\right) .
\end{array}
$$

Let $C=\left\{x \in V_{1} \mid G_{2 x}^{\prime}\right.$ is connected $\}$ and $D=\{y \in$ $V_{2} \mid G_{1 y}^{\prime}$ is connected $\}$.

Throughout this section, we always assume that $G_{1}$ and $G_{2}$ have $m$ and $n$ vertices, respectively, and $\lambda\left(G_{i}\right)=\lambda_{i} \geq 1$ for $i=1,2$. So $\delta\left(G_{i}\right) \geq 1$ for $i=1,2$, which implies $m \geq 2$ and $n \geq 2$.

Lemma 2. $G=G_{1} \times G_{2}$ is a $\lambda^{\prime}$-graph if $m \geq 2$ and $n \geq 2$. 
Proof. Because $m \geq 2$ and $n \geq 2$, the graph $G=G_{1} \times$ $G_{2}$ has $\left|V\left(G_{1} \times G_{2}\right)\right|=m n \geq 4$ vertices, and thus $G$ is not $K_{3}$. Moreover, $\delta(G)=\delta\left(G_{1}\right)+\delta\left(G_{2}\right) \geq 2$, and thus $G$ is not a star. Therefore, $G$ is a $\lambda^{\prime}$-graph.

Theorem 1. $\quad \lambda^{\prime}\left(G_{1} \times G_{2}\right) \geq \min \left\{m \lambda_{2}, n \lambda_{1}, \lambda_{1}+2 \lambda_{2}, 2 \lambda_{1}+\right.$ $\left.\lambda_{2}\right\}$ if $m \geq 3$ and $n \geq 3$.

Proof. Denote $\mu=\min \left\{m \lambda_{2}, n \lambda_{1}, \lambda_{1}+2 \lambda_{2}, 2 \lambda_{1}+\lambda_{2}\right\}$. By Lemma $2, G_{1} \times G_{2}$ is a $\lambda^{\prime}$-graph, so its super edge-cuts always exist. Assume $F$ is a minimum super edge-cut with $|F|<\mu$. We need to show that $G_{1} \times G_{2}-F$ is connected. Because $|F|<\mu \leq m \lambda_{2}$, there exists some $x_{0} \in V_{1}$ such that $G_{2 x_{0}}^{\prime}$ is connected. Because $|F|<\mu \leq n \lambda_{1}$, there exists some $y_{0} \in V_{2}$ such that $G_{1 y_{0}}^{\prime}$ is connected. That is to say, $|C| \geq 1$ and $|D| \geq 1$. There are three cases to be considered for us.

CASE 1. $|C|=1$. This implies that the other $m-1$ subgraphs $G_{2 x}^{\prime}$ are disconnected, where $x \in V_{1} \backslash\left\{x_{0}\right\}$. In this case, $G_{1 y}^{\prime}$ is connected for any $y \in V_{2}$. Otherwise, because $m \geq 3$, we have $|F| \geq(m-1) \lambda_{2}+\lambda_{1} \geq 2 \lambda_{2}+\lambda_{1} \geq \mu$, a contradiction. Thus, $|D|=n$. By Remark $1,\left(\cup_{y \in D} V_{1 y}\right) \cup V_{2 x_{0}}=V_{1} \times V_{2}$ is nonseparable and, thus, $\left(\cup_{y \in D} G_{1 y}^{\prime}\right) \cup G_{2 x_{0}}^{\prime}$ is connected by Lemma 1 and so is $G_{1} \times G_{2}-F$.

CASE 2. $|C|=m$. Because $\left(\cup_{x \in C} V_{2 x}\right) \cup V_{1 y_{0}}=V_{1} \times V_{2}$ is nonseparable, we have $\left(\cup_{x \in C} G_{2 x}^{\prime}\right) \cup G_{1 y_{0}}^{\prime}$ is connected by Lemma 1, which means $G_{1} \times G_{2}-F$ is connected.

CASE 3. $2 \leq|C| \leq m-1$. When $|D|=1$ or $|D|=n$, the connectedness of $G_{1} \times G_{2}-F$ can be derived in the same way as Case 1 or Case 2. Now assume $2 \leq|D| \leq n-1$. On the other hand, $|D| \geq n-1$ because $|C| \leq m-1$, otherwise $|F| \geq$ $2 \lambda_{1}+\lambda_{2} \geq \mu$, a contradiction. Thus, $|D|=n-1$. Similarly, $|C|=m-1$. Without loss of generality, assume $G_{2 x^{\prime}}^{\prime}$ and $G_{1 y^{\prime}}^{\prime}$ are disconnected for some $x^{\prime} \in V_{1}$ and $y^{\prime} \in V_{2}$. That is, $G_{2 x}^{\prime}$ is connected for any $x \neq x^{\prime}$ and $G_{1 y}^{\prime}$ is connected for any $y \neq y^{\prime}$. Because $\left(\cup_{y \neq y^{\prime}} V_{1 y}\right) \cup\left(\cup_{x \neq x^{\prime}} V_{2 x}\right)=V_{1} \times V_{2} \backslash\left\{\left(x^{\prime}, y^{\prime}\right)\right\}$ is nonseparable, by Lemma $1,\left(\cup_{y \neq y^{\prime}} G_{1 y}^{\prime}\right) \cup\left(\cup_{x \neq x^{\prime}} G_{2 x}^{\prime}\right)$ is connected. Because $F$ is a super edge-cut, the vertex $\left(x^{\prime}, y^{\prime}\right)$ is adjacent to $\left(\cup_{y \neq y^{\prime}} G_{1 y}^{\prime}\right) \cup\left(\cup_{x \neq x^{\prime}} G_{2 x}^{\prime}\right)$. So $G_{1} \times G_{2}-F$ is connected and the proof is complete.

Remark 2. The lower bound given above is tight. For example, let $G_{1}=K_{m}$ with the vertex-set $\left\{x_{1}, x_{2}, \ldots, x_{m}\right\}$ and let $G_{2}=K_{1, n-1}$ with the vertex-set $\left\{y_{1}, y_{2}, \ldots, y_{n}\right\}$, where $m \geq 3$ and $n \geq 3$. Then $\lambda_{1}=m-1$ and $\lambda_{2}=1$. By Theorem $1, \lambda^{\prime}\left(G_{1} \times G_{2}\right) \geq \min \left\{m \lambda_{2}, n \lambda_{1}, \lambda_{1}+2 \lambda_{2}, 2 \lambda_{1}+\right.$ $\left.\lambda_{2}\right\}=\min \{m, n(m-1), m+1,2 m-1\}=m$. In addition, if $y_{1} y_{2} \in E\left(G_{2}\right)$, the edge-set $\left\{\left(x_{1}, y_{1}\right)\left(x_{1}, y_{2}\right),\left(x_{2}, y_{1}\right)\left(x_{2}, y_{2}\right)\right.$, $\left.\ldots,\left(x_{m}, y_{1}\right)\left(x_{m}, y_{2}\right)\right\}$ is an edge-cut that isolates no vertex of $K_{m} \times K_{1, n-1}$. So it is a super edge-cut, which implies $\lambda^{\prime}\left(K_{m} \times K_{1, n-1}\right) \leq m$. Therefore, $\lambda^{\prime}\left(K_{m} \times K_{1, n-1}\right)=m$. The lower bound is attained.

Lemma 3 (Hellwig and Volkmann [5]). If G is a $\lambda^{\prime}$-optimal graph, then $\lambda(G)=\delta(G)$.
With the proof of Theorem 4.1 in [11], we obtain the super edge-connectivity of the Cartesian product of two $\lambda^{\prime}$-optimal graphs.

Theorem 2. $\quad \lambda^{\prime}\left(G_{1} \times G_{2}\right)=\min \left\{m \delta\left(G_{2}\right), n \delta\left(G_{1}\right), \xi\left(G_{1} \times\right.\right.$ $\left.\left.G_{2}\right)\right\}$ if $G_{1}$ and $G_{2}$ are both $\lambda^{\prime}$-optimal.

Proof. Denote $\delta\left(G_{i}\right)=\delta_{i}, \lambda\left(G_{i}\right)=\lambda_{i}, \xi\left(G_{i}\right)=\xi_{i}$, $\lambda^{\prime}\left(G_{i}\right)=\lambda_{i}^{\prime}$ for $i=1,2$ and $G=G_{1} \times G_{2}$. Because $G_{i}$ is $\lambda^{\prime}$-optimal, $G_{i}$ is a $\lambda^{\prime}$-graph for $i=1,2$, which implies $m \geq 4, n \geq 4$.

By Lemma $2, \lambda^{\prime}(G)$ is well defined. First, we have $\lambda^{\prime}(G) \leq$ $\xi(G)$ by (1). Because $m \geq 4, E_{G}\left(V_{1} \times\{y\}\right)$ is a super edgecut for a vertex $y \in V_{2}$ with $d_{G_{2}}(y)=\delta_{2}$. So $\lambda^{\prime}(G) \leq m \delta_{2}$. Analogously, we have $\lambda^{\prime}(G) \leq n \delta_{1}$. Thus,

$$
\lambda^{\prime}(G) \leq \min \left\{m \delta_{2}, n \delta_{1}, \xi(G)\right\} .
$$

Assume $\lambda^{\prime}(G)<\min \left\{m \delta_{2}, n \delta_{1}, \xi(G)\right\}$. Let $F$ be a $\lambda^{\prime}$-cut. We should show that $G_{1} \times G_{2}-F$ is connected to deduce a contradiction. Because $|F|<m \delta_{2}=m \lambda_{2}$ by Lemma 3, there exists some $x_{0} \in V_{1}$ such that $G_{2 x_{0}}^{\prime}$ is connected. Analogously, there exists some $y_{0} \in V_{2}$ such that $G_{1 y_{0}}^{\prime}$ is connected. That is to say, $|C| \geq 1$ and $|D| \geq 1$. There are three cases to be considered.

CASE 1. $|C|=m$. By Remark $1,\left(\cup_{y \in D} V_{1 y}\right) \cup\left(\cup_{x \in C} V_{2 x}\right)=$ $V_{1} \times V_{2}$ is nonseparable. Then $\left(\cup_{y \in D} G_{1 y}^{\prime}\right) \cup\left(\cup_{x \in C} G_{2 x}^{\prime}\right)$ is connected by Lemma 1 , and so is $G_{1} \times G_{2}-F$.

CASE 2. $|C|=m-1$. There is only $x_{1} \in V_{1}$ such that $G_{2 x_{1}}^{\prime}$ is disconnected. By Remark 1, $\left(\cup_{y \in D} V_{1 y}\right) \cup\left(\cup_{x \in C} V_{2 x}\right) \stackrel{=}{=}$ $V_{1} \times V_{2}-\left\{\left(x_{1}, y\right) \mid y \in V_{2} \backslash D\right\}$ is nonseparable, and thus by Lemma 1, $\left(\cup_{y \in D} G_{1 y}^{\prime}\right) \cup\left(\cup_{x \in C} G_{2 x}^{\prime}\right)$ is connected. To prove that $G_{1} \times G_{2}-F$ is connected, we only need to show that every vertex in $V^{\prime}=\left\{\left(x_{1}, y\right) \mid y \in V_{2} \backslash D\right\}$ is connected to $\left(\cup_{y \in D} G_{1 y}^{\prime}\right) \cup\left(\cup_{x \in C} G_{2 x}^{\prime}\right)$. To this end, let $\left(x_{1}, y\right)$ be any vertex in $V_{2 x_{1}}(y \notin D)$. Note that $F$ is a super edge-cut. If $\left(x_{1}, y\right)$ is an isolated vertex of $G_{2 x_{1}}^{\prime}$, then it is adjacent to $\left(x^{\prime}, y\right)\left(x^{\prime} \neq\right.$ $\left.x_{1}\right)$, which is in $\left(\cup_{y \in D} G_{1 y}^{\prime}\right) \cup\left(\cup_{x \in C} G_{2 x}^{\prime}\right)$. In the following, we suppose that $\left(x_{1}, y\right)$ is contained in a component of $G_{2 x_{1}}^{\prime}$ with at least two vertices. Denote this component by $H$. We only need to consider the case of $V(H) \subseteq V^{\prime}$, otherwise $\left(x_{1}, y\right)$ is connected to $\left(\cup_{y \in D} G_{1 y}^{\prime}\right) \cup\left(\cup_{x \in C} G_{2 x}^{\prime}\right)$. There are two subcases to be considered.

SUBCASE 2.1. $G_{2 x_{1}}-V(H)$ contains a component with at least two vertices, denoted by $H^{\prime}$. Because $G_{2 x_{1}}$ is connected, all the components of $G_{2 x_{1}}-V(H)$ different from $H^{\prime}$, if any, are connected to $H$ and not connected to $H^{\prime}$. So $G_{2 x_{1}}-$ $V\left(H^{\prime}\right)$ is connected with $\left|V\left(G_{2 x_{1}}-V\left(H^{\prime}\right)\right)\right| \geq|V(H)| \geq$ 2, which implies that $E_{G_{2 x_{1}}}\left(V\left(H^{\prime}\right)\right)$ is a super edge-cut. Hence, we conclude that $\left|F \cap E\left(G_{2 x_{1}}\right)\right| \geq\left|E_{G_{2 x_{1}}}(V(H))\right| \geq$ $\left|E_{G_{2 x_{1}}}\left(V\left(H^{\prime}\right)\right)\right| \geq \lambda_{2}^{\prime}$. There is at least one vertex in $V(H)$ with neighbors in $\left(\cup_{y \in D} G_{1 y}^{\prime}\right) \cup\left(\cup_{x \in C} G_{2 x}^{\prime}\right)$. Otherwise, we obtain the following contradiction

$$
|F| \geq \lambda_{2}^{\prime}+|V(H)| \delta_{1} \geq \lambda_{2}^{\prime}+2 \delta_{1}=\xi_{2}+2 \delta_{1} \geq \xi(G) .
$$

So the vertex $\left(x_{1}, y\right)$ is connected to $\left(\cup_{y \in D} G_{1 y}^{\prime}\right) \cup\left(\cup_{x \in C} G_{2 x}^{\prime}\right)$. 
SUBCASE 2.2. $G_{2 x_{1}}-V(H)$ contains only isolated vertices. Then $\left|F \cap E\left(G_{2 x_{1}}\right)\right| \geq\left|E_{G_{2 x_{1}}}(V(H))\right| \geq(n-|V(H)|) \delta_{2}$. Let $\Delta_{2}$ be the maximum degree of $G_{2}$. Obviously, $\Delta_{2} \leq n-1$ and $\xi_{2} \leq \Delta_{2}+\delta_{2}-2$. We claim that there is at least one vertex in $V(H)$ with neighbors in $\left(\cup_{y \in D} G_{1 y}^{\prime}\right) \cup\left(\cup_{x \in C} G_{2 x}^{\prime}\right)$. Otherwise,

$$
\begin{aligned}
|F| & \geq(n-|V(H)|) \delta_{2}+|V(H)| \delta_{1} \\
& =\delta_{2}+(n-|V(H)|-1) \delta_{2}+(|V(H)|-2) \delta_{1}+2 \delta_{1} \\
& \geq \delta_{2}+(n-|V(H)|-1)+|V(H)|-2+2 \delta_{1} \\
& =\delta_{2}+(n-3)+2 \delta_{1} \\
& \geq \delta_{2}+\Delta_{2}-2+2 \delta_{1} \\
& \geq \xi_{2}+2 \delta_{1} \\
& \geq \xi(G),
\end{aligned}
$$

a contradiction. Therefore, the vertex $\left(x_{1}, y\right)$ is connected to $\left(\cup_{y \in D} G_{1 y}^{\prime}\right) \cup\left(\cup_{x \in C} G_{2 x}^{\prime}\right)$.

In a word, $G_{1} \times G_{2}-F$ is connected in this case.

CASE 3. $|C| \leq m-2$. Similarly, $\left(\cup_{y \in D} G_{1 y}^{\prime}\right) \cup\left(\cup_{x \in C} G_{2 x}^{\prime}\right)$ is connected. In this case, to prove that $G_{1} \times G_{2}-F$ is connected, we only need to show that every vertex of $G_{2 x}^{\prime}$ is connected to $\left(\cup_{y \in D} G_{1 y}^{\prime}\right) \cup\left(\cup_{x \in C} G_{2 x}^{\prime}\right)$ for $x \notin C$.

Suppose that $G_{2 x}^{\prime}$ contains a component with at least two vertices, denoted by $H_{x}$, which has no vertices in $\left(\cup_{y \in D} G_{1 y}^{\prime}\right) \cup\left(\cup_{x \in C} G_{2 x}^{\prime}\right)$ for $x \notin C$. If $G_{2 x}-V\left(H_{x}\right)$ contains a component with at least two vertices, similar to Subcase 2.1, we have $\left|F \cap E\left(G_{2 x}\right)\right| \geq\left|E_{G_{2 x}}\left(V\left(H_{x}\right)\right)\right| \geq \lambda_{2}^{\prime}$. Hence, $|D| \geq n-1$, otherwise, noting that $G_{1}$ is $\lambda^{\prime}$-optimal, by Lemma 3,

$|F| \geq\left|F \cap E\left(G_{2 x}\right)\right|+2 \lambda_{1} \geq \lambda_{2}^{\prime}+2 \delta_{1}=\xi_{2}+2 \delta_{1} \geq \xi(G)$,

a contradiction. The case $|D| \geq n-1$ can be handled in the same way as Case 1 and Case 2 .

If $G_{2 x}-V\left(H_{x}\right)$ contains only isolated vertices, then $\mid F \cap$ $E\left(G_{2 x}\right)|\geq| E_{G_{2 x}}\left(V\left(H_{x}\right)\right) \mid \geq\left(n-\left|V\left(H_{x}\right)\right|\right) \delta_{2}$. Because $H_{x}$ has no vertices in $\left(\cup_{y \in D} G_{1 y}^{\prime}\right) \cup\left(\cup_{x \in C} G_{2 x}^{\prime}\right)$ by our assumption,

$$
\begin{aligned}
|F| & \geq\left(n-\left|V\left(H_{x}\right)\right|\right) \delta_{2}+\left|V\left(H_{x}\right)\right| \lambda_{1} \\
& =\left(n-\left|V\left(H_{x}\right)\right|\right) \delta_{2}+\left|V\left(H_{x}\right)\right| \delta_{1} \quad(\text { by Lemma 3) } \\
& =\delta_{2}+\left(n-\left|V\left(H_{x}\right)\right|-1\right) \delta_{2}+\left(\left|V\left(H_{x}\right)\right|-2\right) \delta_{1}+2 \delta_{1} \\
& \geq \delta_{2}+\left(n-\left|V\left(H_{x}\right)\right|-1\right)+\left|V\left(H_{x}\right)\right|-2+2 \delta_{1} \\
& =\delta_{2}+(n-3)+2 \delta_{1} \\
& \geq \delta_{2}+\Delta_{2}-2+2 \delta_{1} \\
& \geq \xi_{2}+2 \delta_{1} \\
& \geq \xi(G),
\end{aligned}
$$

where $\Delta_{2}$ is the maximum degree of $G_{2}$, a contradiction. So, every vertex of $H_{x}$ is in or connected to $\left(\cup_{y \in D} G_{1 y}^{\prime}\right) \cup$ $\left(\cup_{x \in C} G_{2 x}^{\prime}\right)$.
Suppose that vertex $(x, y)(y \notin D)$ is isolated in $G_{2 x}^{\prime}$. Then it is not isolated in $G_{1 y}^{\prime}$, otherwise, it is isolated in $G_{1} \times G_{2}-F$, contradicting our hypothesis that $F$ is a super edge-cut. So the vertex $(x, y)$ is contained in a component with at least two vertices of $G_{1 y}^{\prime}$. We can show that vertex $(x, y)$ is connected to $\left(\cup_{y \in D} G_{1 y}^{\prime}\right) \cup\left(\cup_{x \in C} G_{2 x}^{\prime}\right)$ in the same way as above.

Because all possible cases lead to a contradiction, $\lambda^{\prime}(G)=$ $\min \left\{m \delta_{2}, n \delta_{1}, \xi(G)\right\}$ and the proof is complete.

From Theorem 2, we can easily obtain the following corollary.

Corollary 1 (Ueffing and Volkmann [11]). Let $G_{1}$ and $G_{2}$ be two disjoint $\lambda^{\prime}$-optimal graphs and let $G=G_{1} \times G_{2}$. Then $G$ is $\lambda^{\prime}$-optimal or the $\lambda^{\prime}$-atoms of $G$ have the form $\{x\} \times V_{2}$ for a vertex $x \in V_{1}$ with $d_{G_{1}}(x)=\delta\left(G_{1}\right)$ or $V_{1} \times\{y\}$ for a vertex $y \in V_{2}$ with $d_{G_{2}}(y)=\delta\left(G_{2}\right)$.

The $n$-dimensional toroidal mesh $C\left(d_{1}, d_{2}, \ldots, d_{n}\right)$ ([12]) can be expressed as the Cartesian product $C_{d_{1}} \times C_{d_{2}} \times \cdots \times$ $C_{d_{n}}$, where $C_{d_{i}}$ is a cycle of length $d_{i}$ for $i=1,2, \ldots, n$.

Corollary 2 (Xu and Xu [16]). Let $C\left(d_{1}, d_{2}, \ldots, d_{n}\right)$ be the $n$-dimensional toroidal mesh. Then $\lambda^{\prime}\left(C\left(d_{1}, d_{2}, \ldots, d_{n}\right)\right)=$ $4 n-2$ and, thus, $C\left(d_{1}, d_{2}, \ldots, d_{n}\right)$ is $\lambda^{\prime}$-optimal if $d_{i} \geq 4$ for each $i=1,2, \ldots, n$.

Proof. We prove the corollary by induction on $n$. It is easy to see that a cycle $C_{d_{i}}$ is $\lambda^{\prime}$-optimal for $d_{i} \geq 4$. Now we assume $n \geq 2$. Suppose $G_{n-1}=C\left(d_{1}, d_{2}, \ldots, d_{n-1}\right)$ is $\lambda^{\prime}$ optimal, which implies $\lambda^{\prime}\left(G_{n-1}\right)=4(n-1)-2=4 n-6=$ $\xi\left(G_{n-1}\right) .\left|V\left(G_{n-1}\right)\right|=d_{1}+d_{2}+\cdots+d_{n-1}$ and $\lambda\left(G_{n-1}\right)=$ $2(n-1)$. Denote $G=C\left(d_{1}, d_{2}, \ldots, d_{n}\right)=G_{n-1} \times C_{d_{n}}$. Noting $d_{i} \geq 4$ for each $i=1,2, \ldots, n$, by Theorem 2 , we have

$$
\begin{aligned}
\lambda^{\prime}(G) & =\min \left\{\left|V\left(G_{n-1}\right)\right| \delta\left(C_{d_{n}}\right),\left|V\left(C_{d_{n}}\right)\right| \delta\left(G_{n-1}\right), \xi(G)\right\} \\
& =\min \left\{2\left(d_{1}+d_{2}+\cdots+d_{n-1}\right), 2 d_{n}(n-1), 4 n-2\right\} \\
& =4 n-2=\xi(G) .
\end{aligned}
$$

Thus, $G$ is $\lambda^{\prime}$-optimal.

Theorem 3. Let $G_{0}$ be a connected graph with $n$ vertices and $\lambda\left(G_{0}\right)=\lambda$. Then $\lambda^{\prime}\left(K_{2} \times G_{0}\right)=\min \{n, 2 \lambda\}$.

Proof. Let $V\left(G_{0}\right)=\left\{v_{1}, v_{2}, \ldots, v_{n}\right\}$ and $G=K_{2} \times$ $G_{0}$. By the definition of the Cartesian product, $K_{2} \times G_{0}$ is obtained from two copies of $G_{0}$ by connecting (via a new edge) vertex $v_{i}$ in one copy to the vertex $v_{i}$ in the other copy of $G_{0}, 1 \leq i \leq n$. These new edges are called cross edges. Denote the two copies by $G_{1}$ and $G_{2}$, respectively, and let $V_{1}=V\left(G_{1}\right), V_{2}=V\left(G_{2}\right)$.

If $\left|V\left(G_{0}\right)\right| \geq 2$, then $E_{G}\left(V_{1}\right)$ is a super edge-cut of $G$, and hence, $\lambda^{\prime}(G) \leq\left|E_{G}\left(V_{1}\right)\right|=\left|V_{1}\right|=n$. Suppose $X_{1} \subseteq V_{1}$ with $d_{G_{1}}\left(X_{1}\right)=\lambda$ and let $X_{2} \subseteq V_{2}$ be the set of those vertices adjacent to $X_{1}$. It is easy to see $E_{G}\left(X_{1} \cup X_{2}\right)$ is a super edge-cut 
of $G$, and hence, $\lambda^{\prime}(G) \leq\left|E_{G}\left(X_{1} \cup X_{2}\right)\right|=2\left|E_{G_{1}}\left(X_{1}\right)\right|=2 \lambda$. It follows that

$$
\lambda^{\prime}(G) \leq \min \{n, 2 \lambda\} .
$$

We will show that the equality in (2) holds. Suppose to the contrary that $\lambda^{\prime}(G)<\min \{n, 2 \lambda\}$. We will show $G-F$ is connected for any $\lambda^{\prime}$-cut $F$ of $G$.

Let $F$ be a $\lambda^{\prime}$-cut of $G$. Denote $G_{1}^{\prime}=G_{1}-F$ and $G_{2}^{\prime}=G_{2}-$ $F$. Since $|F|<2 \lambda$, at least one of $G_{1}^{\prime}$ and $G_{2}^{\prime}$ is connected. Without loss of generality, assume $G_{2}^{\prime}$ is connected. If $G_{1}^{\prime}$ is also connected, then $G_{1}^{\prime}$ is connected to $G_{2}^{\prime}$ by one cross edge because $|F|<n$. Hence, $G-F$ is connected.

In the following we suppose that $G_{1}^{\prime}$ is not connected. For any vertex $v_{i}$ of $G_{1}^{\prime}$, denote the crossedge incident with it by $e$. If $e \notin F$, then $v_{i}$ is connected to $G_{2}^{\prime}$. If $e \in F$, then $v_{i}$ is adjacent to other vertices of $G_{1}^{\prime}$ as $F$ is a super edgecut. Denote the component of $G_{1}^{\prime}$ containing $v_{i}$ by $G_{11}$ and $X=V\left(G_{11}\right)$. Let $t=|X|$. Clearly, $t \geq 2$. If some vertex of $G_{11}$ is connected to $G_{2}^{\prime}$ in $G-F$, then the vertex $v_{i}$ is also connected to $G_{2}^{\prime}$. Otherwise, $|F| \geq d_{G_{1}}(X)+|X| \geq \lambda+t$. Because $|F|<2 \lambda$, we have $t<2 \lambda-\lambda=\lambda \leq \delta$. That is to say, $t \leq \delta-1$ and $\delta \geq 3$. Every vertex in $X$ has at least $\delta-(t-1)$ neighbors in $V_{1} \backslash X$, so

$$
|F| \geq d_{G_{1}}(X)+t \geq(\delta-(t-1)) t+t=-t^{2}+(\delta+2) t .
$$

Define a function $f(t)=-t^{2}+(\delta+2) t$. It is easy to see the function $f(t)$ reaches the minimum value at an end-point of the interval $[2, \delta-1]$. Because $f(2)=2 \delta$ and $f(\delta-1)=$ $3 \delta-3=2 \delta+(\delta-3) \geq 2 \delta$, we obtain a contradiction that $|F| \geq 2 \delta \geq 2 \lambda$. Therefore, the equality in (2) follows.

Corollary 3 (Li and Xu [7]). Let $G_{0}$ be a connected graph of order $n(\geq 2)$. Then

$$
\lambda^{\prime}\left(K_{2} \times G_{0}\right)=\min \left\{n, 2 \delta\left(G_{0}\right), 2 \lambda^{\prime}\left(G_{0}\right)\right\} .
$$

Proof. If $G_{0}$ is super edge-connected, $\lambda\left(G_{0}\right)=\delta\left(G_{0}\right)<$ $\lambda^{\prime}\left(G_{0}\right)$. If $G_{0}$ is not super edge-connected, then $\lambda\left(G_{0}\right)=$ $\lambda^{\prime}\left(G_{0}\right)$. Noting that $\lambda\left(G_{0}\right) \leq \delta\left(G_{0}\right)$, we have $\min \left\{\delta\left(G_{0}\right)\right.$, $\left.\lambda^{\prime}\left(G_{0}\right)\right\}=\lambda\left(G_{0}\right)$. Thus, $\min \left\{\delta\left(G_{0}\right), \lambda^{\prime}\left(G_{0}\right)\right\}=\lambda\left(G_{0}\right)$ for any connected graph $G_{0}$, and thus the corollary holds by Theorem 3 .

Corollary 4 (Esfahanian [2]). Let $Q_{n}$ be an n-dimensional cube. Then $\lambda^{\prime}\left(Q_{n}\right)=2 n-2$ and, thus, $Q_{n}$ is $\lambda^{\prime}$-optimal for $n \geq 2$, and is super- $\lambda$ for $n \geq 3$.

Proof. Because $Q_{n}=K_{2} \times Q_{n-1}$ (see Section 3.1 in [12]), by Theorem $3, \lambda^{\prime}\left(Q_{n}\right)=\min \left\{\left|V\left(Q_{n-1}\right)\right|, 2 \lambda\left(Q_{n-1}\right)\right\}=$ $\min \left\{2^{n-1}, 2 n-2\right\}=2 n-2=\xi\left(Q_{n}\right)$ and thus $Q_{n}$ is $\lambda^{\prime}$ optimal for $n \geq 2$. In addition, $\lambda^{\prime}\left(Q_{n}\right)=2 n-2>\lambda\left(Q_{n}\right)=n$ for $n \geq 3$, so $Q_{n}$ is super- $\lambda$.

Combining Theorem 1 with Theorem 3, we obtain the main result in $[1,10]$.

Corollary 5. Assume $G_{1} \times G_{2} ¥ K_{2} \times K_{n}$ for $n \geq 2$. If $G_{i}$ are regular and max- $\lambda$ for $i=1,2$, then $G_{1} \times G_{2}$ is super $-\lambda$.
Proof. Note that $\lambda\left(G_{1} \times G_{2}\right) \leq \delta\left(G_{1} \times G_{2}\right)=d_{1}+d_{2}$, where $d_{i}$ is the regular degree of $G_{i}$ for $i=1,2$. Let $m=$ $\left|V\left(G_{1}\right)\right|, n=\left|V\left(G_{2}\right)\right|$ and $\lambda_{i}=\lambda\left(G_{i}\right)$ for $i=1,2$.

When $m \geq 3$ and $n \geq 3$, we have $d_{i}>1$ for $i=1,2$ and

$$
\begin{aligned}
m \lambda_{2} \geq\left(d_{1}+1\right) \lambda_{2} & =\left(d_{1}+1\right) d_{2}=d_{1} d_{2}+d_{2}>d_{1}+d_{2} \\
n \lambda_{1} \geq\left(d_{2}+1\right) \lambda_{1} & =\left(d_{2}+1\right) d_{1}=d_{1} d_{2}+d_{1}>d_{1}+d_{2} \\
\lambda_{1}+2 \lambda_{2} & =d_{1}+2 d_{2}>d_{1}+d_{2} \\
2 \lambda_{1}+\lambda_{2} & =2 d_{1}+d_{2}>d_{1}+d_{2}
\end{aligned}
$$

When $m=2$, we have $d_{1}=1$ and $1<d_{2}<n-1$ (because $G_{1} \times G_{2} \nsucceq K_{2} \times K_{n}$ ), and

$$
\begin{aligned}
& n>1+d_{2}=d_{1}+d_{2} \\
& 2 \lambda_{2}=2 d_{2}>d_{1}+d_{2} .
\end{aligned}
$$

By Theorem 1 and Theorem 3, the corollary holds.

\section{Acknowledgment}

The authors thank the anonymous referees for their helpful comments and suggestions.

\section{REFERENCES}

[1] W.S. Chiue and B.S. Shieh, On connectivity of the Cartesian product of two graphs, Appl Math Comput 102 (1999), 129-137.

[2] A.H. Esfahanian, Generalized measures of fault tolerance with application to $n$-cube networks, IEEE Trans Comput 38 (1989), 1586-1591.

[3] A.H. Esfahanian and S.L. Hakimi, On computing a conditional edge-connectivity of a graph, Inform Process Lett 27 (1988), 195-199.

[4] M.A. Fiol, J. Fábrega, and M. Escudero, Short paths and connectivity in graphs and digraphs, Ars Combin 29B (1990), 253-256.

[5] A. Hellwig and L. Volkmann, Sufficient conditions for graphs to be $\lambda^{\prime}$-optimal, super-edge-connected, and maximally edgeconnected, J Graph Theory 48 (2005), 228-246.

[6] L. Lesniak, Results on the edge-connectivity of graphs, Discrete Math 8 (1974), 351-354.

[7] L. Li and J.M. Xu, On restricted edge-connectivity of vertex-transitive graphs, J China Univ Sci Tech 34 (2004), 266-272.

[8] M. Lü and J.M. Xu, Super connectivity of line graphs and digraphs, Acta Math Appl Sinica (English Series) 22 (2006), 43-48.

[9] J.X. Meng and Y.H. Ji, On a kind of restricted edge connectivity of graphs, Discrete Appl Math 117 (2002), 183-193.

[10] B.S. Shieh, Super edge- and point-connectivities of the Cartesian product of regular graphs, Networks 40 (2002), 91-96. 
[11] N. Ueffing and L. Volkmann, Restricted edge-connectivity and minimum edge-degree, Ars Combin 66 (2003), 193203.

[12] J.M. Xu, Topological structure and analysis of interconnection networks, Kluwer Academic Publishers, Dordrecht, 2001.

[13] J.M. Xu, Theory and application of graphs, Kluwer Academic Publishers, Dordrecht, 2003.

[14] J.M. Xu and M. Lü, On restricted edge-connectivity of regular digraphs, Taiwan J Math 9 (2005), 661-670.
[15] J.M. Xu, M. Lü, M.J. Ma, and A. Hellwig, Super connectivity of line graphs, Inform Process Lett 94 (2005), 191-195.

[16] J.M. Xu and K.L. Xu, On restricted edge-connectivity of graphs, Discrete Math 243 (2002), 291-298.

[17] C.S. Yang, J.F. Wang, J.Y. Lee, and F.T. Boesch, Graph theoretic reliability analysis for the Boolean $n$-cube networks, IEEE Trans Circuits Syst 35 (1988), 1175-1179.

[18] C.S. Yang, J.F. Wang, J.Y. Lee, and F.T. Boesch, The number of spanning trees of the regular networks, Int J Comput Math 23 (1988), 185-200. 